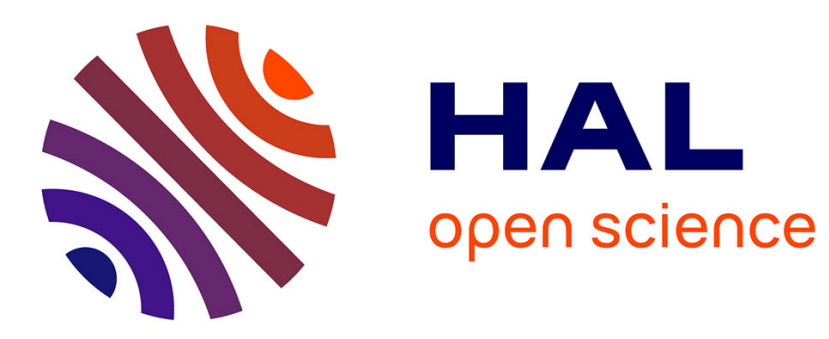

\title{
Photoionization time delays
}

J M Dahlström, Morgane Vacher, Alfred Maquet, Jérémie Caillat, Stefan Haessler

\section{To cite this version:}

J M Dahlström, Morgane Vacher, Alfred Maquet, Jérémie Caillat, Stefan Haessler. Photoionization time delays. Markus Kitzler; Stefanie Gräfe. Ultrafast Dynamics Driven by Intense Light Pulses, Springer, 2016, 978-3-319-20172-6. hal-01164799

\section{HAL Id: hal-01164799 https://hal-ensta-paris.archives-ouvertes.fr/hal-01164799}

Submitted on 17 Jun 2015

HAL is a multi-disciplinary open access archive for the deposit and dissemination of scientific research documents, whether they are published or not. The documents may come from teaching and research institutions in France or abroad, or from public or private research centers.
L'archive ouverte pluridisciplinaire HAL, est destinée au dépôt et à la diffusion de documents scientifiques de niveau recherche, publiés ou non, émanant des établissements d'enseignement et de recherche français ou étrangers, des laboratoires publics ou privés. 


\section{Photoionization time delays}

J. Marcus Dahlström ${ }^{1,2}$, Morgane Vacher $^{3}$, Alfred Maquet ${ }^{4,5}$, Jérémie Caillat $^{4,5}$, and Stefan Haessler ${ }^{6,7}$

1 Department of Physics, Stockholm University, AlbaNova University Center, SE-10691 Stockholm, Sweden

2 Max-Planck Institute for the Physics of Complex Systems, Noethnitzerstr. 38, 01187 Dresden, Germany

3 Department of Chemistry, Imperial College London, London, SW7 2AZ, United Kingdom

4 UPMC, UMR 7614, Laboratoire de Chimie Physique - Matière et Rayonnement 11, rue Pierre et Marie Curie, 75231 Paris Cedex 05, France

${ }^{5}$ CNRS, UMR 7614, Laboratoire de Chimie Physique - Matière et Rayonnement 11, rue Pierre et Marie Curie, 75231 Paris Cedex 05, France

6 Photonics Institute, Vienna University of Technology, Gußhausstraße 27/387, 1040 Vienna, Austria

7 Laboratoire d'Optique Appliqueé, ENS de Techniques Avancées-Paristech, Ecole Polytechnique, CNRS, UMR7639, 91761 Palaiseau Cedex, France

Abstract. The material presented in this chapter is based on important advances realized in "attophysics" which make feasible to follow the motion of electrons in atoms and molecules with attosecond-level time resolution. In this context, timedelays have been recently determined in the process of photoionization by extremeultra-violet (XUV) pulses and the question of the significance of these measured delays arises. As we shall outline here, numerical experiments show that they are intimately related to the structure of the ionized species' continuous spectrum. Another point addressed here is that, in experiments, the measurements have the common characteristic to be performed in the presence of an auxiliary infra-red (IR) field, used to "clock" the timing of the process. This implies to adapt the theory treatment to handle such "two-color" photoionization processes. We review a systematic analysis of these features that are characteristic of this class of electronic transitions, when viewed in the time domain.

Wednesday $18^{\text {th }}$ February, 2015, 12:04

\subsection{Introduction}

"Attophysics" has emerged in the 2000s with the advent of a new generation of radiation sources delivering attosecond pulses of Extreme Ultra-Violet (XUV) radiation via the High-order Harmonic Generation (HHG) process [1-3]. 
These new sources allow the photoionization process to be considered as taking place at a well defined time within a brief time window with attosecond resolution. This opens up the possibility to achieve the real-time probing and control of electron (or hole) dynamics on a sub-femtosecond time scales $[4,5]$. It is in this very active context that several different experiments have evidenced attosecond time-delays that are associated with the photoionization process [6-12]. Complementary interpretations of these experiments has been reviewed within the frameworks of both the time-independent [13] and the time-dependent picture [14].

This set of results have been obtained thanks to the advances realized in both the attosecond metrology and generation schemes of XUV pulses through HHG. One has led to the generation of isolated pulses with duration down to 80 as, [15] while the other has resulted in the emission of attosecond pulse trains, with bursts of XUV radiation having durations down to 63 as [16]. When recombining such XUV pulses with an infrared (IR) laser radiation, one can realize two-color pump-probe experiments, with sub-femtosecond temporal resolution. One important outcome has been to study the photoionization of atoms in the time domain, thus evidencing intrinsic time-delays associated with the process [6,9-11]. As we shall show, the physical significance of the delays so measured depends on the electronic structure of the excited species, including the influence of resonant states. Regarding the input of attophysics in this latter field, c.f. refs. [17-19]

Before to proceed, we wish to make clear the distinction existing between the attosecond scale relevant to intra-atomic electronic transitions and the much longer time scale governing the detection of the wave packet created in the ionization process. When the atom is irradiated by an attosecond XUV pulse, the width of the energy content of the photoionized wave packet is governed by the time-energy uncertainty principle. While traveling over a macroscopic distance towards the detector, the motion of the maximum of the density probability is essentially classical. In parallel, all energy components of the electron wave packet (EWP) will spread so that the spatio-temporal structure also reaches the macroscopic realm. This allows for direct detection of the electron energy distribution as a function of time-of-flight to the detector. The important point is that, although the electronic transition is taking place on an attosecond scale, in the presence of the IR clock a signature of the time-development of the process can be extracted from the photoelectron spectrum.

The organization of the chapter is as follows: In sec. 1.2, we will follow the wave packet evolution using time-dependent simulations and extract the delays due to the microscopic interactions that have occurred in the target. The results are directly compared with theoretical delays obtained from timeindependent scattering theory. In sec. 1.3 we provide an interpretation of the microscopic delays in photoionization using a formalism designed to compute two-photon (XUV-IR) matrix elements. In sec. 1.4 we give a review of experi- 
mental work on photoionization delays using two-color (XUV-IR) fields. Finally, in sec. 1.5 we present our conclusions.

\subsection{Phase-shifts and time-delays}

\subsubsection{Formal definition of a photoionization delay}

The characterization of the dynamics of a photoelectron wave packet commonly refers to the notion of scattering time-delay developed in the context of quantum collision theory [20,21]. A wave packet scattered by a potential experiences a delay as compared to free motion. Wigner and his collaborators $[20,21]$ first pointed out that this delay can be characterized through the spectral derivative of a phase shift $\eta$ :

$$
\tau=\hbar \frac{\partial \eta}{\partial E}
$$

where $E$ represents the particle's energy ${ }^{1}$. A stationary-phase derivation shows that this delay, evaluated at the energy of maximum amplitude, represents the delay induced by the potential on the traveling particle, compared to the time it takes a reference particle (typically a free particle) with the same energy to cover the same distance: In Eq. 1.1, it is implicit that $\eta$ is the phase difference (or phase shift) between the scattered and the reference waves ${ }^{2}$. It must be clear also that the reference has to be chosen to match the scattering wave in the incoming region (usually far from the interaction region). Moreover, since a collision treated quantum mechanically is described with the help of wave packets, its dynamics can never be completely represented by a unique delay: The group delays are representative quantities in principle only for near Fourier-limited, ideally Gaussian distributions.

Extending Wigner's formalism to photoionization processes is straightforward if one admits that a photoelectron experiences a "half collision" as it is ejected, while remaining under the influence of the ionic core [13]. Further, a group delay can be assigned to the spectral phase that the photoelectron wave packet accumulates during and upon the transition. This comes in addition to the imprint of the driving electric field phase exploited in standard XUV pulse characterization techniques such as RABBIT [24,25] or FROG-CRAB [26].

\section{Scattering delay}

In the case of one-photon ionization, the only contribution to the phase of the EWP is associated with the real-valued continuum wave function selected by

\footnotetext{
${ }^{1}$ Other formal definitions can be invoked, see Ref. [22] for a comprehensive review.

2 The stationary-phase approximation is discussed in e.g. Ref. [23]
} 
the transition [27]. Just as for collisions, that phase is actually a phase shift, the definition of which comes with the choice of a reference.

When taking the free-particle as a reference, the overall scattering phase associated with photoionization decomposes as the sum of a short-range and a long-range contributions,

$$
\eta=\eta_{\mathrm{SR}}+\eta_{\mathrm{LR}}
$$

The long-range term, $\eta_{\mathrm{LR}}$, is due to the asymptotic Coulomb tail associated with any atomic or molecular ionic core potential and it depends logarithmically on the electron position. The short-range term, $\eta_{\mathrm{SR}}$, contains a signature of the detailed interactions between the released electron and the remaining ones which constitute the ionic core, as well as the multi-center nuclear structure in the case of molecules.

According to Eq. 1.2, the group delay associated with scattering upon photoionization reads as the sum

$$
\underbrace{\frac{\partial \eta}{\partial E}}_{\tau_{\mathrm{sca}}}=\underbrace{\frac{\partial \eta_{\mathrm{SR}}}{\partial E}}_{\tau_{\mathrm{SR}}}+\underbrace{\frac{\partial \eta_{\mathrm{LR}}}{\partial E}}_{\tau_{\mathrm{LR}}}
$$

of a long-range delay $\tau_{\mathrm{LR}}$ and a short-range delay $\tau_{\mathrm{SR}}$. While $\tau_{\mathrm{LR}}$ is everywhere modified by the infinite-range influence of the Coulomb potential, $\tau_{\mathrm{SR}}$ converges after some finite distance representative of the size of the atom or the molecule, i.e. beyond the influence of the short-range component of the potential. As already mentioned, the implicit reference in Eq. 1.2 and 1.3 is the free motion.

Note that for practical reasons, scattering phase shifts and Wigner-like delays are usually assigned to angular momentum components of the complete EWP expanded on a partial wave basis. Partial-wave decompositions are essential in atomic calculations as they allow the use of angular momentum theory. Formal links between the Wigner delay, the lifetime matrix, $Q$, and the scattering matrix, $S$, were first derived by Smith [21]. In the present context, such constructions provide a way to separate the total wave packet into partial wave packets in different ionic channels. The dynamics of each of these can be properly characterized by a simple Wigner-like group delay.

Besides, when choosing specific representations of the continuum, the scattering phase shift sometimes manifests as a contribution to the phase of a complex valued wave-function. This occurs for example when treating the continuum with conventional scattering waves, initially developed in the $S$ matrix formalism to represent a full collision. This phase then shows up as the argument of the associated (otherwise real-valued) transition matrix element: One indeed often refers to the scattering phase shift as the "phase of the dipole matrix element". Similarly, it was shown recently that the one-photon dipole matrix element becomes complex if correlation effects are included perturbatively on a basis of uncorrelated single particle function products [28]. 


\section{Beyond 1-photon transitions}

The fact that the real-valued dipole operator itself does not bring any contribution to the phase of the released EWP reflects the instantaneous nature of single photon absorption (or its annihilation in the quantumelectrodynamics formalism). However, in multiphoton transitions associated with above-threshold ionization (ATI), i.e. when intermediate states lie in the continuous spectrum, the transition operator (typically the Green operator in a 2-photon transition) becomes complex, thus bringing an additional contribution to the spectral phase of the released wave packet.

Following the line of reasoning developed for the scattering delay, this additional phase can be used in the time domain to introduce a "transition delay" $\tau_{\text {tra }}$ accounting for the temporal shaping of the EWP as it builds up during the transition [29]. In particular, it can be interpreted as a transition duration when the energy transferred by one of the photons coincides with the excitation energy towards a resonant state possessing a significant lifetime $\tau_{\mathrm{R}}$ : $\tau_{\text {tra }}$ is then related to $\tau_{\mathrm{R}}$ and represents the time spent by the system in this intermediate resonance before transiting toward the final state ${ }^{3}$. Experimentally, accessing this "transition phase" requires an interferometric setup with two paths leading coherently to the same final continuum state. These requirements are met by the RABBIT technique [31], a method initially designed to characterize attosecond XUV pulse trains using an IR pulse as an external clock $[24,25,32,33]$. This technique was thus revisited in $[7,8,29,34]$ to investigate XUV-IR 2-color photoionization in the time-domain, where two distinct 2-photon transitions probe each other.

Furthermore, it was shown that the phase of the transition matrix element in two-photon XUV-IR ionization through an intermediate continuum is related to the short-range scattering phase shift $\left(\eta_{\mathrm{SR}}\right)$ of the intermediate state $[10,13,35,36]$. It was verified numerically for hydrogen that the intermediate scattering phase could be recovered with high fidelity if a universal phase shift, called the continuum-continuum phase, was subtracted from the two-photon matrix element [36]. This reinterpretation of RABBIT measurements [10], which states that the one-photon ionization delays can be experimentally obtained, has now been verified by diagrammatic methods for both neon and argon atoms [37]. In this reinterpretation, the IR pulse is seen as a probe used to characterize the dynamics of XUV-driven 1-photon ionization ${ }^{4}$. The RABBIT setup shares fundamental similarities with the streaking technique $[14,36,38]$, which (i) also consists of HHG-based XUV-pump IR-probe schemes, (ii) was initially conceived to characterize XUV pulses (using a FROGCRAB analysis), and (iii) had already been revisited to access photoionization delays [9]. The main difference lies in the pulse properties: XUV pulse train

\footnotetext{
${ }^{3}$ See $e g[30]$ for a comprehensive derivation and exploitation of transitions delays in the particular context of resonant X-ray Raman scattering.

${ }^{4}$ Note that the two reinterpretations of RABBIT in terms of transition or scattering delays are not contradictory, but rather complementary.
} 
and weak IR field in RABBIT versus broad, single XUV pulse and moderate IR field for streaking. Section 1.3 of the present chapter is dedicated in more details to the specific subject of probing one-photon ionization delays using dressing IR fields, but first we turn to time-domain simulations of microscopic delays and compare the results with scattering theory.

\subsubsection{Ionization dynamics in numerical experiments}

The physical relevance of the scattering delay as defined above can be addressed in simulations of photoionization, where the actual dynamics observed in numerical experiments is compared to the delays evaluated from energydependent scattering phase shifts, using Wigner's formalism. To this end, we used simple models of atoms allowing extensive numerical experiments where the formal delays are compared to the exact (numerical) solution of the timedependent Schrödinger equation (TDSE).

\section{The model atoms}

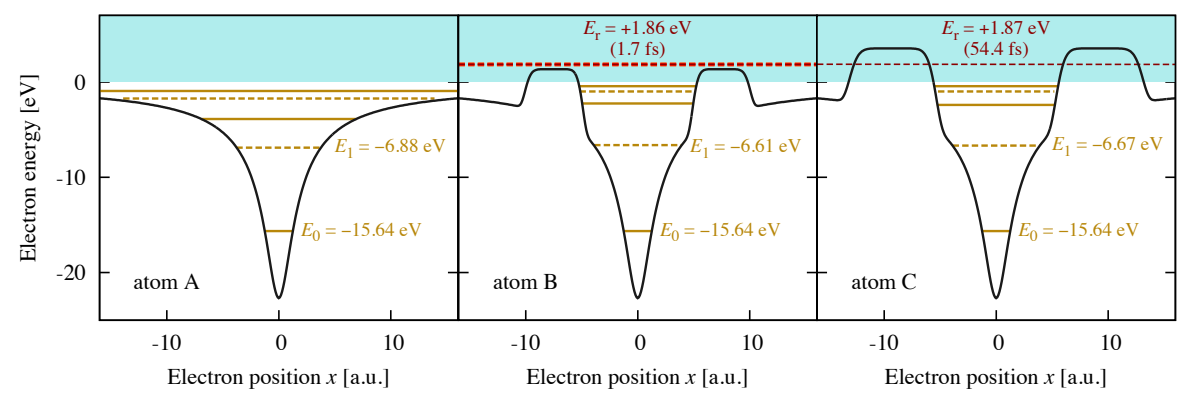

Fig. 1.1. Model potentials used in our simulations. The potential for model atom A (left) consists in a simple soft-Coulomb term. The potentials for model atoms $\mathrm{B}$ (center) and $\mathrm{C}$ (right) contain additional barriers inducing structures in the continuum. Horizontal lines represent discrete energy levels: bound states $(E<0$, yellow) and resonances $(E>0$, red). The line styles indicate the parity of each state: even (full) or odd (dashed). The energy (and life-time) of the states relevant for our simulations are also indicated.

Our generic model consists in a single active electron (with position $x$ ) interacting with an effective core through a symmetric potential in one dimension. We will consider three different potentials defining three different systems: A, B and C. Atom A is built on a simple soft-Coulomb potential ${ }^{5}$,

$$
V(x)=-\frac{1}{\sqrt{x^{2}+a^{2}}}
$$

\footnotetext{
${ }^{5}$ The equations are displayed in atomic units: $\hbar=m=e=1 /\left(4 \pi \epsilon_{0}\right)=1$.
} 
where the regularizing parameter $a$ is the main knob to control the energies of the bound states. The potentials for atoms B and C read

$$
V(x)=-\frac{1}{\sqrt{x^{2}+a^{2}}}+G(x)
$$

where $G(x)$ represents two symmetric hyper-gaussian barriers, see Fig. 1.1. In such models, the short-range barriers are adjusted to design shape resonances, accounting qualitatively for the mean-field interaction between the active electron and the frozen cores. The spectral positions of the shape resonances depend mainly on the barrier separation, and their widths on the height and width of the barriers.

Note that because we use symmetric potentials, the discrete bound states wave-functions are alternately even and odd (starting with an even wavefunction for the ground state) whereas degenerate even and odd continuum states are found for any positive energy. This property is of first importance in the simulations since a dipole transition between two such states is allowed only if their parity is opposite, allowing for a direct treatment of the continuum in terms of real-valued wave-functions $[27,29,39]$, with defined parities. In the following, we denote $\phi_{E, p}(x)$ the continuum eigenfunction associated with the positive energy $E$ and parity $p(+1$ and -1 for an even and odd parity respectively). The potentials were adjusted to create a relatively broad, odd resonance near threshold in atom $\mathrm{B}$, and a narrower one, also odd, in atom $\mathrm{C}$ (see Fig. 1.1).

The time evolution of the system's wave-function $\psi(x, t)$ follows the timedependent Schrödinger equation:

$$
i \frac{\partial}{\partial t} \psi(x, t)=\left[-\frac{1}{2} \frac{\partial^{2}}{\partial x^{2}}+i A(t) \frac{\partial}{\partial x}+V(x)\right] \psi(x, t),
$$

where the interaction with light is treated in the velocity gauge $(A \cdot p)$ representation of the dipole approximation. We solved it numerically using the Crank-Nicolson algorithm [40]. The temporal dependence of the vector potential $A(t)$ is defined by a carrier frequency $\omega_{0}$ and a generic $\sin ^{2}$-envelope centered at $t=0$, with a duration of 100 cycles FWHM. In the range of considered photon energies $\left(15.8 \mathrm{eV}<\omega_{0}<18.8 \mathrm{eV}\right)$, it represents pulse durations of $\sim 25 \mathrm{fs}$, which lies between the lifetimes of the resonances in atom B (1.7 $\mathrm{fs})$ and in atom $\mathrm{C}(54.4 \mathrm{fs})$. The Xuv pulse intensities were kept low enough so that non-linear effects could be safely neglected.

\section{1-photon transition dynamics and scattering delay}

We present here results illustrating the relevance and limitations of the formal scattering delay (Eq. 1.3) as a characteristic time of 1-photon ionization dynamics. More precisely, our study focuses on the short-range contribution 

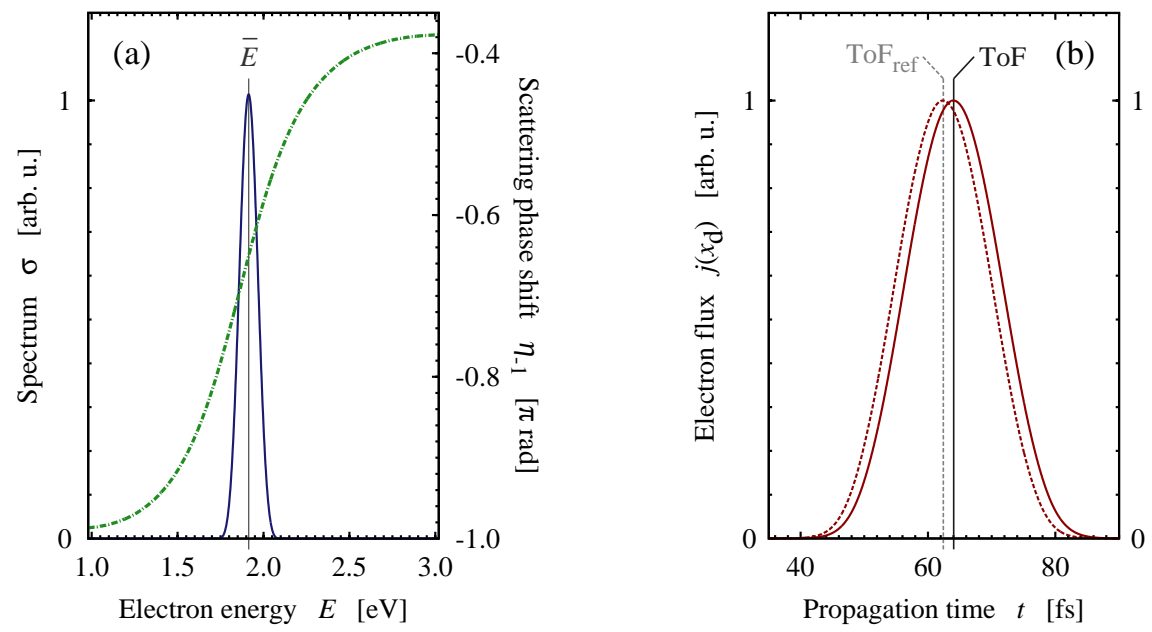

Fig. 1.2. Model atom B (ground state) @ 17.55 eV - (a) full blue curve: electron spectrum (the vertical line indicates the mean energy under the peak $\bar{E}$ ); dash-dotted green curve: phase shift of the odd scattering state (atom A as a reference); (b) full red curve: electron flux $j\left(x_{\mathrm{d}}\right)$ evaluated at $x_{\mathrm{d}}=1000$ a.u. versus propagation time; dashed red curve: $j\left(x_{\mathrm{d}}\right)$ evaluated at the same distance when photoionizing the reference atom $\mathrm{A}$ with a pulse adjusted to obtain the same average energy $\bar{E}$; vertical lines: average times under the peaks, ToF (full) and ToF ref (dashed) respectively. For a better readability, the spectrum and flux data were normalized to 1 at their maximum values.

to the delays, $\tau_{\mathrm{SR}}$ : we thus investigated the photoionization dynamics of atoms $\mathrm{B}$ and $\mathrm{C}$, taking atom $\mathrm{A}$ as a reference (see Fig. 1.1).

We first detail the procedure for a near resonant ionization of atom B, where the resonance is short-lived enough to induce negligible distortions on the bell-shaped temporal and spectral profiles, but where the delay is large enough to be visualized on the wave packet temporal profile. We present afterwards the results obtained near a sharper resonance and in a smooth continuum.

\section{Near a broad resonance}

Our analysis consists in merging temporal and spectral information retrieved from photoelectron wave packets. Figure 1.2(a) displays the spectral profile $\sigma(E)$ of the wave packet created by ionizing atom B initially in its ground state, with a pulse of central energy $\omega_{0}=17.55 \mathrm{eV}$. The spectrum was retrieved by using the spectral analysis technique described in Ref. [41]. The wave packet is centered close to the resonant energy $\left(E_{\mathrm{R}}=1.87 \mathrm{eV}\right)$, within its width $\left(\Gamma_{\mathrm{R}}=0.383 \mathrm{eV}\right)$. The associated mean photoelectron energy computed as 

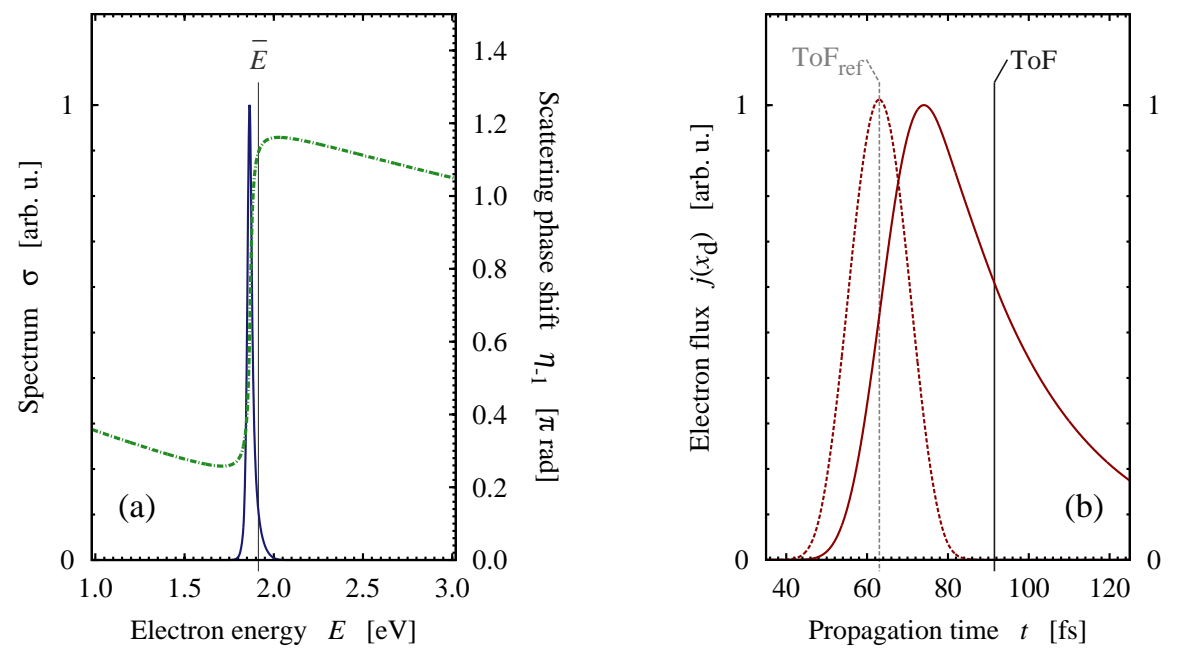

Fig. 1.3. Model atom C (ground state) @ $\mathbf{1 7 . 5 5} \mathbf{~ e V ~ - ~ ( a ) ~ f u l l ~ b l u e ~ c u r v e : ~}$ electron spectrum (the vertical line indicates the mean energy under the peak $\bar{E}$ ); dash-dotted green curve: phase shift of the odd scattering state (atom A as a reference); (b) full red curve: electron flux $j\left(x_{\mathrm{d}}\right)$ evaluated at $x_{\mathrm{d}}=1000$ a.u. versus propagation time; dashed red curve: $j\left(x_{\mathrm{d}}\right)$ evaluated at the same distance when photoionizing the reference atom $A$ with a pulse adjusted to obtain the same average energy $\bar{E}$; vertical lines: average times under the peaks, ToF (full) and ToF ref (dashed) respectively. For a better readability, the spectrum and flux data were normalized to 1 at their maximum values.

$$
\bar{E}=\frac{1}{P_{\text {ion }}} \int_{\text {peak }} E \times \sigma(E) \mathrm{d} E
$$

where $P_{\text {ion }}$ is the total ionization probability, is equal to $1.86 \mathrm{eV}$. From the temporal point of view, we characterized the wave packet dynamics by computing the electron flux as a function of time at a position $x_{\mathrm{d}}=1000 \mathrm{a} . \mathrm{u}$, beyond the short-range part of the potential, as

$$
j\left(x_{\mathrm{d}}, t\right)=\operatorname{Im}\left\{\left[\psi\left(x_{\mathrm{d}}, t\right)\right]^{\star} \psi^{\prime}\left(x_{\mathrm{d}}, t\right)\right\}
$$

where $\psi^{\prime}$ is the first order derivative of $\psi$ with respect to $x$ and * stands for complex conjugate. From the flux, shown in Fig. 1.2(b), we defined a numerical "time-of-flight" as the average arrival time at the virtual detector,

$$
\operatorname{ToF}\left(x_{\mathrm{d}}\right)=\frac{1}{P_{\text {ion }}} \int_{\text {peak }} t \times j\left(x_{\mathrm{d}}, t\right) \mathrm{d} t .
$$

In the considered case, it amounts to 64.04 fs. That average value is indeed close to the time it would take a free particle with a kinetic energy of 1.86 $\mathrm{eV}$ to travel the distance of 1000 a.u (65.42 fs), and the difference evidences 

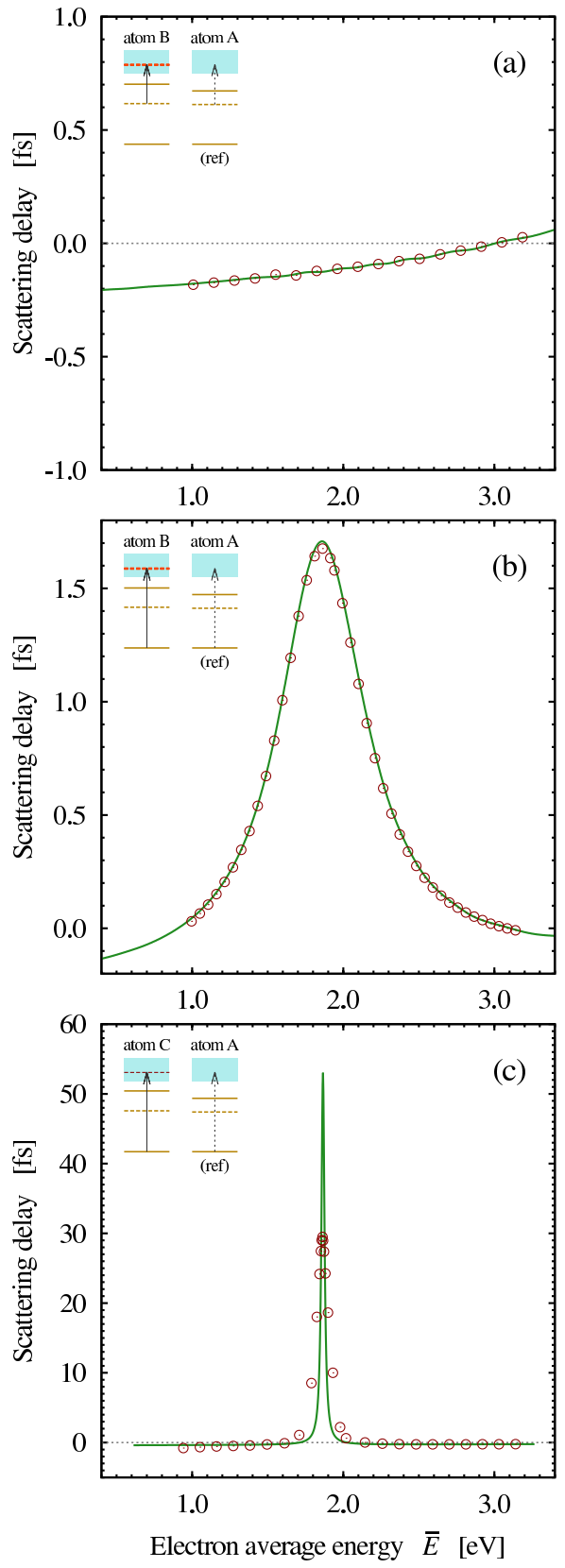

Fig. 1.4. Scattering delays evaluated as a time-of flight difference (circles) and as a formal group delay (full curves), as a function of the mean photoelectron energy $\bar{E}$ (see text). (a) smooth continuum: ionization of atom B compared to ionization of atom A, both from their first excited state (the resonance is transparent the transition, for parity reasons); (b) broad resonance: ionization of atom B compared to ionization of atom A, both from their ground state; (c) sharp resonance: ionization of atom $\mathrm{C}$ compared to ionization of atom $\mathrm{A}$, both from their ground state. 
the influence of the potential on the electron motion, all along the way to the detector.

To reveal the role of the short-range barriers on the release dynamics, and more specifically on the ToF value, we simulated the photoionization of the reference atom A (with the interaction potential restricted to the soft Coulomb term) with a central pulse frequency finely adjusted to create a wave packet with the same average energy $\bar{E}=1.86 \mathrm{eV}$. The flux computed at $x_{\mathrm{d}}$ for this reference transition presents a temporal profile similar to the near resonant transition in atom B (also displayed in Fig. 1.2(b)). It is however clearly shifted towards shorter times: The associated average time-of-flight, ToF ref, is equal to $62.40 \mathrm{fs}$. The time-of-flight difference

$$
\Delta \mathrm{TOF}=\operatorname{ToF}\left(x_{\mathrm{d}}\right)-\operatorname{ToF}_{\text {ref }}\left(x_{\mathrm{d}}\right)
$$

is equal to $1.64 \mathrm{fs}$. It is a numerical evaluation of the delay induced on the released wave packet by the short-range barriers in atom B, seen from a timedependent perspective, to be compared to the formal scattering delay.

We thus numerically computed the real-valued continuum eigenfunctions selected by the transition [39] $\phi_{E, p}(x)$ for atom $\mathrm{B}$ and $\phi_{E, p}^{\mathrm{ref}}(x)$ for the referenceatom $\mathrm{A}$ and finally determined their relative phase shift $\eta_{p}(E)$. The phase shift $\eta_{-1}(E)$ computed in the energy range covered by the wave packet is shown as a dashed curve in Figure 1.2(a). It undergoes a smooth $\simeq 0.6 \pi$ jump spread over the resonance width. Its spectral derivative evaluated at the average energy $\bar{E}$, i.e. the formal scattering delay $\tau_{\text {sca }}$, is equal to $1.65 \mathrm{fs}$, which is remarkably close to the value of $\Delta$ ToF.

The procedure was then repeated for a set of pulse frequencies near the resonance. Figure 1.4(b) compares the values of $\Delta$ ToF (circles) obtained in the time-dependent treatment (Eq. $1.8-1.10)$, to the group delays $\tau_{\text {sca }}$ (full line) derived from the scattering phase shifts and evaluated at the mean wave packet energies $\bar{E}$. The two sets of data follow a typical bell-shaped curve, with a maximum at resonance reaching the resonance lifetime $\Gamma_{\mathrm{R}}^{-1}=1.7 \mathrm{fs}$. They are in very good agreement with the most noticeable discrepancy at resonance $(\bar{E} \simeq 1.87 \mathrm{eV})$, i.e. where the wave packet distorsion is expected to be maximum.

\section{Near a narrow resonance}

The situation is different when the transition reaches the vicinity of a resonance narrower than the pulse spectral width. A representative case is given by the resonant ionization of atom $\mathrm{C}$ taken in its ground state.

In the wave packet launched by a $\hbar \omega_{0}=17.55 \mathrm{eV}$ pulse, the resonance now manifests as a sharp, prominent, peak distorting the otherwise smooth, bell-shaped spectral profile, as displayed in Figure 1.3(a). Furthermore, the electron flux shown in Fig. 1.3(b) (still computed at $x_{\mathrm{d}}=1000$ a.u.), now displays an asymmetric tail towards larger times - a direct signature of the 
resonance's exponential decay. At this point, it is already clear that such a structured wave packet cannot be accurately characterized by a single energy $(e g \bar{E})$, nor its elongated temporal profile by a single time (eg ToF). Taking again atom $\mathrm{A}$ as a reference provides a time of flight difference $\Delta$ TоF $=$ $28.46 \mathrm{fs}$, while the formal delay derived from the scattering phase shift $\eta_{-1}(E)$ (shown in Figure 1.3(a)) at the average energy $\bar{E}$ is above $50 \mathrm{fs}$.

We again repeated the simulations by changing the central frequency, and reported the delays in Figure 1.4(c). Although both sets of data display the same peaked shape centered at the resonant energy $E_{\mathrm{R}}=1.86 \mathrm{eV}$, they present clear discrepencies. In particular, the maximum value reached by $\Delta$ ToF is approximately twice smaller than the actual resonance lifetime and the $\Delta$ ToF peak is broadened by the light pulse width, as compared to the $\tau_{\text {sca }}$ peak.

\section{In a smooth continuum}

The formal scattering delay, validated before as representative of the ionization dynamics near a broad resonance, is expected to be just as relevant in a smooth continuum. To illustrate this, we simulated photoionizations of atom $\mathrm{B}$, now initially considered in its first excited state with odd symmetry. The pulse central frequency was varied to end up in the same electron energy region, between 1 and $3 \mathrm{eV}$. Selection rules are such that now, the odd resonance is transparent to one-photon transitions, for parity reasons.

We followed the same time-energy analysis as for the two previous cases. The delays, computed as the time-of-flight difference $\Delta$ ToF on the one hand and as the phase shift derivative $\tau_{\text {sca }}$ on the other hand, are compared in Figure 1.4(a). The delay evolves monotonically, and the two sets of data are indeed in very good agreement.

The results presented above illustrate the validity of the formal scattering delay as representative of the average one-photon ionization dynamics, at least when the created wave packets are unstructured enough both in the energy and time domains. We mention that the significance and relevance of the formal transition delay can be evidenced in a similar manner in two-photon ionization simulations $[29,34]$.

\subsection{Analysis of two-photon XUV +IR ionization}

A real-world time-of-flight spectometer for detecting photoelectrons operates on the basis of separating different energy (or momentum) components through macroscopic propagation $[42,43]$. Indeed, this constitutes a frequency domain measurement and information about microscopic time-delays cannot be resolved. However, if the photoionization process is modulated by an IR laser field, it is possible to encode some temporal information into the spectral domain by construction of a so-called spectrogram over photoelectron kinetic energy (or momentum) and sub-cycle delay between XUV and IR 
fields $[26,31,44,45]$. This is done by repeating the experiment as a function of the delay between XUV and IR fields. Still, timing information about the one-photon process is not directly recorded and further theoretical calibration is required, as we shall briefly discuss in this section.

In the presence of an IR laser field, the photoelectron released upon absorption of one XUV photon can further absorb and emit IR photons, thereby changing its energy. Provided that the IR field is weak, the energy of the electron will change as the result of the exchange of only one IR photon at most, so that the overall process amounts to a two-photon transition. These measurements are based on the interference of several multiphoton pathways that lead to the same final continuum state. The contribution from the final state scattering phase cancels out and the so-called transition phase through the different pathways will determine the observed delay in the measurement.

In the following, we will restrict the analysis to ATI in a smooth continuum where the photoelectron interacts perturbatively with both the XUV and IR fields through second-order perturbation theory. The two-photon process induced by the linearly polarized field, $E(\omega)$, is expressed as an S-matrix:

$$
S_{f i}^{(2)}=\frac{1}{2 \pi i} \int d \omega^{\prime} M_{f i}^{(2)}\left(\omega^{\prime}\right) E\left(\omega^{\prime}\right) E\left(\omega^{\prime \prime}\right),
$$

where the two-photon matrix element from the initial state $|i\rangle$ (with energy $\epsilon_{i}$ ) and to the final state $|f\rangle$ (with energy $\epsilon_{f}$ ) is

$$
M_{f i}^{(2)}\left(\omega^{\prime}\right)=\lim _{\varepsilon \rightarrow 0^{+}} \sum_{n} \frac{\langle f|z| n\rangle\langle n|z| i\rangle}{\left(\epsilon_{i}+\omega^{\prime}-\epsilon_{n}+i \varepsilon\right)},
$$

with $\omega^{\prime}$ being the energy of the first absorbed photon and where the integralsum runs over intermediate states $n$ (with energy $\epsilon_{n}$ ). The integral over $\omega^{\prime}$ in Eq. (1.11) can be seen as a sum over all quantum paths that lead from the initial state to the final state with the energy-conservation requirement: $\omega^{\prime}+\omega^{\prime \prime}=\epsilon_{f}-\epsilon_{i}$. In our two-color case, the field has two different components at XUV and IR frequencies for the pump and probe fields, respectively. The dominant contribution to Eq. (1.11) can then identified as the process with absorption of one XUV photon followed by exchange of one IR photon. If both components have a broad bandwidth then there is a broad range of photonpairs available. However, the effects of this convolution are negligible if one of the fields is quasi-monochromatic. We will assume that the IR field is quasimonochromatic with frequency $\omega_{\mathrm{IR}}=\omega$ and with a bandwith $\Delta_{\mathrm{IR}}$ that is narrower than the bandwidth of the XUV field with frequencies $\Omega_{>}$and $\Omega_{<}$. The two dominant quantum paths become:

$$
\begin{aligned}
S_{f i}^{(a)} & \approx \frac{1}{2 \pi i} M_{f i}^{(2)}\left(\Omega_{<}\right) E\left(\Omega_{<}\right) E(\omega) \Delta_{\mathrm{IR}} \\
S_{f i}^{(e)} & \approx \frac{1}{2 \pi i} M_{f i}^{(2)}\left(\Omega_{>}\right) E\left(\Omega_{>}\right) E^{*}(\omega) \Delta_{\mathrm{IR}}
\end{aligned}
$$




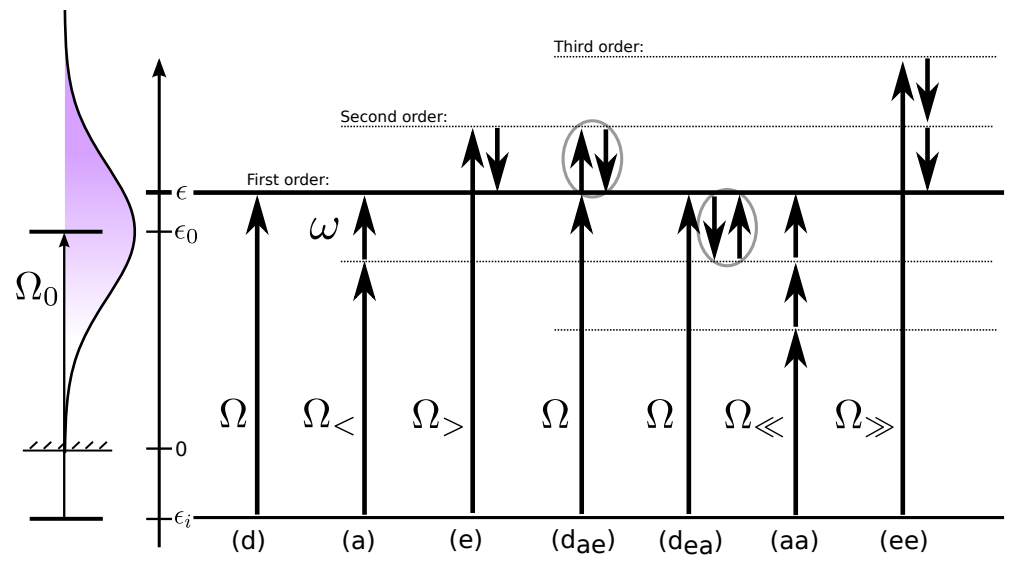

Fig. 1.5. Photon-diagrams for laser-assisted photoionization: Single XUV-photon absorption contributes with a direct path (d) to the final state. The dominant twophoton processes involve the absorption or emission of a laser photon indicated by paths (a) and (e). Higher-order processes involve the exchange of more laser photons. Figure adapted from Ref. [35].

corresponding to absorption (a) and emission (e) of a laser photon from the fields as depicted in Fig. 1.5. Note that the intermediate XUV photon energy is different in the two paths to ensure global energy conservation. For this class of multiphoton processes numerically exact computations can only be performed in one-electron (hydrogen-like) and two-electron (helium-like) systems. In single-ionization events the wave packet can be described as a single excited electron at a large radial distance far from the ionic core. Within the single-active electron approximation, it is feasible to derive a convenient approximation method designed to evaluate the time delay induced by the IR-driven transition in the continuum. It has been demonstrated that the main results from this analysis also hold for the delay in photoionization from many-electron atomic systems $[37,46]$.

\subsubsection{Asymptotic approximation for ATI transition amplitudes}

In order to obtain an estimate for the phase of the two-photon matrix element, we will rely on an approximation, which utilizes the asymptotic continuum forms of the final state and of the first-order perturbed wavefunction. Specializing to the case of a hydrogenic system with nuclear charge $Z$, the $S$-matrix in Eq. (1.11) can be evaluated approximately, as shown in Ref. [36]. One notices that the phase of the two-photon matrix element contains only phase-shifts that are governed by the angular momentum $\lambda$ of the intermediate state, i.e. a state that can be reached via single-photon ionization. More precisely, for a given transition channel (characterized by the angular momenta of the intermediate and final state: $\ell_{i} \rightarrow \lambda \rightarrow L$ ), the phase of the matrix element 

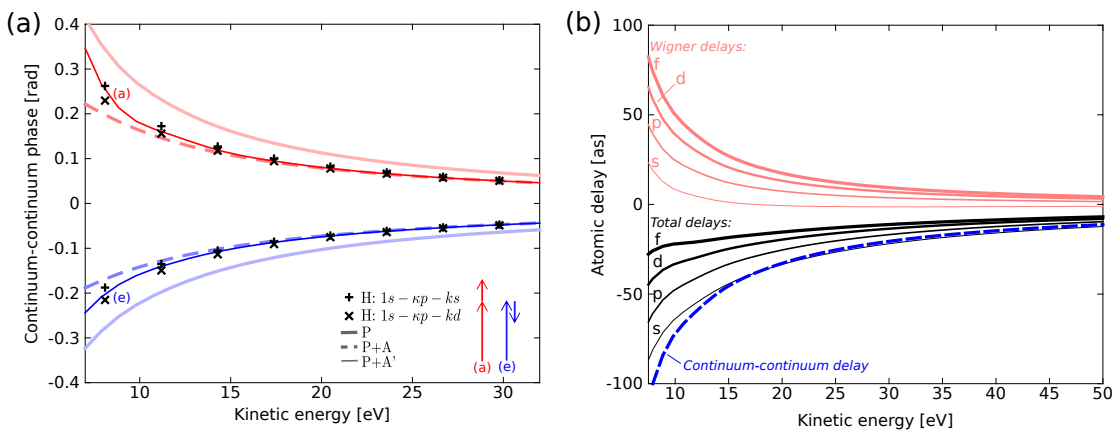

Fig. 1.6. (a) Continuum-continuum phases: ATI phase-shifts for absorption (a) of one $800 \mathrm{~nm}$ laser photon; and for stimulated emission (e) of one laser photon, in a Coulomb potential with $Z=1$. The asymptotic approximation $(\mathrm{P})$ provides the correct qualitative behaviour, while the long-range amplitude corrections $(\mathrm{P}+\mathrm{A}$ and $\mathrm{P}+\mathrm{A}^{\prime}$ ) lead to quantitative agreement, at high enough energy, with the exact calculations in hydrogen $(+$ and $\times)$. (b) Ionization delays: The Wigner delays for hydrogen with a photoelectron of $s, p, d$ or $f$-character [red, upper curves] plus the universal continuum-continuum delay for the laser-probing process with $Z=$ 1 and $\hbar \omega=1.55 \mathrm{eV}$ [blue, dashed curve], yields the total delay in laser-assisted photoionization [black, lower curves]. Figure adapted from Ref. [35].

reduces to:

$$
\begin{aligned}
\arg \left[S_{f i}^{\left(L, \lambda, m_{i}\right)}\right] & =\pi+\arg \left[Y_{L, m_{i}}(\hat{k})\right]+\phi_{<}+\phi \\
& -\frac{\pi \lambda}{2}+\eta_{\lambda}(\kappa)+\phi_{c c}(k, \kappa, Z),
\end{aligned}
$$

where $\phi_{<}$and $\phi$ are the phases of the XUV field, $\Omega_{<}$, and of the IR laser, $\omega$, respectively; and where the continuum-continuum phase is approximated by

$$
\phi_{c c}(k, \kappa, Z) \equiv \arg \left\{\frac{(2 \kappa)^{i Z / \kappa}}{(2 k)^{i Z / k}} \frac{\Gamma[2+i Z(1 / \kappa-1 / k)]+\gamma(k, \kappa)}{(\kappa-k)^{i Z(1 / \kappa-1 / k)}}\right\},
$$

where $k$ and $\kappa$ are the final and intermediate momentum, respectively; and $Z$ is the residual charge of the ion. The first term in Eq. (1.15), which only includes the effects of long-range phase distortion due to the ionic potential, corresponds to the label (P) in Fig. 1.6 (a). The additional term, $\gamma(k, \kappa)$ in Eq. (1.15), is a correction term that arises from amplitude effects due to the ionic potential,

$$
\gamma(k, \kappa)=i Z \frac{(\kappa-k)\left(\kappa^{2}+k^{2}\right)}{2 \kappa^{2} k^{2}} \Gamma[1+i Z(1 / \kappa-1 / k)],
$$

which is shown with the label $(\mathrm{P}+\mathrm{A})$ in Fig. 1.6 (a). The result presented as $\left(\mathrm{P}+\mathrm{A}^{\prime}\right)$ in Fig. (1.6) is a fit to the exact calculation for hydrogen by $\mathrm{R}$. Taïeb [36]. We stress that the final state scattering phase, $\eta_{L}(k)$, cancels out 
and that it does not enter in Eq. (1.14). In the next section we explain how the "delay in photoionization" can be constructed using two-photon matrix elements.

\subsubsection{Extracting time-delay information from laser-assisted photoionization signals}

Having established the asymptotic approximation for the complex amplitudes of ATI processes, we now turn to the probability for the emission of a photoelectron with energy $\epsilon_{k}=\epsilon_{i}+\Omega$, as depicted in Fig. 1.5. The probability is given by the square of the sum of the amplitudes:

$$
\begin{aligned}
P_{\boldsymbol{k}} & \approx\left|S_{d}+S_{a}+S_{e}\right|^{2} \\
& =\left|S_{d}\right|^{2}+\left|S_{a}\right|^{2}+\left|S_{e}\right|^{2} \\
& +2 \Re\left\{S_{d}^{*}\left(S_{a}+S_{e}\right)+S_{a}^{*} S_{e}\right\},
\end{aligned}
$$

where $d, a$ and $e$ label the paths associated with the lowest-order processes: (d), (a) and (e) in Fig. 1.5. The total probability depends on the relative phase of all individual quantum paths. In experiments, the phase of the two-photon amplitudes labelled (a) and (e) can be controlled by changing the sub-cycle delay, $\tau$, between the probe field and the attosecond pulses. More precisely, one controls the relative phase of the IR field as compared to the XUV field, $\phi_{\equiv \omega \tau}$ in Eq. (1.14). The probe-phase dependence is $S_{a} \propto E(\omega) \propto \exp [i \omega \tau]$ and $S_{e} \propto E^{*}(\omega) \propto \exp [-i \omega \tau]$. This implies that the cross-terms in Eq. (1.17) vary differently as a function of $\tau$ : (d)-(a) and (d)-(e) are modulated with periodicity $\omega \tau$ associated with the exchange of only one IR photon [47]; while the cross-term (a)-(e) is modulated with periodicity $2 \omega \tau$ due to the two IR photons involved [31,48]. The maximal probability for photoemission in a RABITT sideband ${ }^{6}$ occurs when the amplitudes associated with paths (a) and (e) are in phase, $\arg \left[S_{a}\right]=\arg \left[S_{e}\right]$, which, using the explicit phases of the relevant two-photon matrix elements in Eq. (1.14) and assuming that an intermediate angular channel $(\lambda)$ is dominant, leads to:

$$
\begin{aligned}
\tau & =\overbrace{\frac{\phi_{\Omega_{>}}-\phi_{\Omega_{<}}}{2 \omega_{r}}}^{\tau_{G D}} \\
& +\underbrace{\frac{\eta_{\lambda}\left(\kappa_{>}\right)-\eta_{\lambda}\left(\kappa_{<}\right)}{2 \omega_{r}}}_{\tau_{\lambda}}+\underbrace{\frac{\phi_{c c}\left(k, \kappa_{>}\right)-\phi_{c c}\left(k, \kappa_{<}\right)}{2 \omega_{r}}}_{\tau_{c c}},
\end{aligned}
$$

\footnotetext{
${ }^{6}$ It has been shown theoretically that the delay of the photoelectron spectrogram in an attosecond streak-camera experiment, as compared to the time variation of the IR vector potential, is equal to the shift of the corresponding RABITT sidebands, c.f. refs $[14,36,38]$.
} 
where the momenta $\kappa_{<}$and $\kappa_{>}$correspond to absorption of an XUV photon $\Omega_{<}$and $\Omega_{>}$, respectively. In Eq. (1.18), we observe that the XUV-IR delay, $\tau$, that maximizes the yield is a sum of three delays:

- $\tau_{G D}$ : the group delay of the XUV field is relative to the subcycle oscillations of the IR probe field.

- $\tau_{\lambda}$ : the Wigner delay is the "delay" in single-photon ionization (must be deduced from the difference with the delay in a reference system, e.g. hydrogen).

- $\tau_{c c}$ : the continuum-continuum delay is a measurement-induced delay from the interplay of the IR field and the long-range Coulomb potential.

The sum: $\tau_{\lambda}+\tau_{c c}=\tau_{\theta}$ constitutes the so-called "atomic delays". Its variation in terms of the kinetic energy of the photoelectron are shown in Fig. 1.6 (b) for the case of hydrogen the angular momentum, $\lambda$. A similar separation of the time-delays as shown in Eq. (1.18) was reported by the group of Burgdörfer for streaking of single attosecond pulses by solving numerically the timedependent Schrödinger equation $[49,50]$. Interestingly, they also showed that $\tau_{c c}$ (there called 'Coulomb-laser coupling') could be calculated from a classical ensemble of electron trajectories.

The delays presented in Eq. (1.18) are calculated from the finite-difference approximations to the actual derivatives, $\tau_{G D}=\partial \phi_{\Omega} / \partial \Omega$ and $\tau_{\lambda}=\partial \eta_{\kappa, \lambda} / \partial \Omega$. For these approximations to be valid, we must require that the spectral phases vary slowly, $\left|\Delta \phi_{\Omega}\right| \equiv\left|\phi_{\Omega>}-\phi_{\Omega_{<}}\right| \ll 2 \pi$, with a small phase variation over the energy range spanned by two laser photons, $\Delta \Omega=2 \omega$. In this sense, "slow" reactions, such as autoionization of a resonance discussed in sec. 1.2.2, require a more detailed evaluation of the spectral integral in Eq. (1.11) [34,51]. In this context, we wish to mention the work that has been made also using the soft-photon approximation (SPA) where Volkov solutions are used as approximate continuum states [52-54]. The advantage of this approximation is that it allows for analytical solutions to non-perturbative IR interactions with the photoelectron, however, because the electron-ion Coulomb interaction is omitted neither the scattering phases nor the continuum-continuum phases will be recovered by SPA. Prior to these successful demonstrations of the seperability of the $\tau_{\lambda}$ and $\tau_{c c}$ in Eq. (1.18), the influence of the short-range scattering phase-shifts was identified by Yakovlev et al [55] and the importance of the long-range Coulomb tail was discussed by Zhang and Thumm [56].

The effects of electron-ion interaction for laser-assisted XUV ionization has also been studied by Smirnova and coworkers using the Eikonal-Volkov Approximation (EVA) [57]. With EVA it was possible to provide accurate results for the photoionization delays from hydrogen [58]. More recently, Eq. (1.18) has been validated, not only for hydrogen, but also for noble-gas atoms by diagrammatic methods [37]; effective two-electron models [50]; and for the $\mathrm{H}_{2}^{+}$ molecule [59]. 

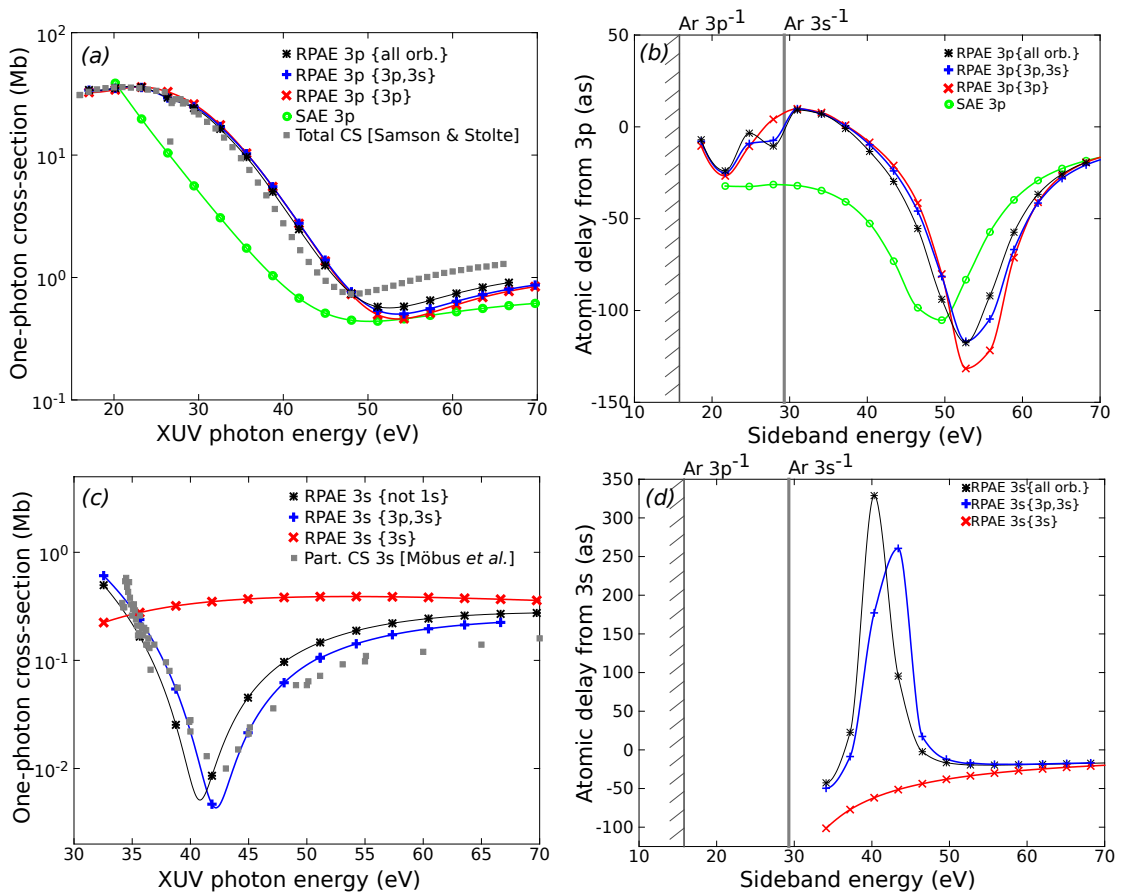

Fig. 1.7. (a) Theoretical partial photoionization cross-section for Ar $3 p^{-1}$ compared to the total photoionization cross-section measurements by Samson and Stolte [60]. (b) Atomic delay for Ar3 $p^{-1}$ observed along the polarization axis of the fields. (c) Theoretical partial photoionization cross-section for $\mathrm{Ar} 3 s^{-1}$ compared to partial cross-section measurements by Möbus et al. [61]. (d) Atomic delay for Ar3s ${ }^{-1}$ observed along the polarization axis of the fields. The correlated orbitals in the RPAE model are listed in the curly brackets. The IR photon is $1.55 \mathrm{eV}$. SAE calculations are performed using the potential proposed by Muller [62]. Figure adapted from Ref. [63].

\section{The delay paradox}

Eq. (1.18) opens up for the possibility to measure (i) $\tau_{\mathrm{GD}}$ of the attosecond pulse or (ii) $\tau_{\lambda}$ of the photoelectron. The former requires exact knowledge of $\tau_{\theta}=\tau_{\lambda}+\tau_{c c}$, which then must be subtracted from the experimental data; while $\tau_{\lambda}$ requires exact knowledge of $\tau_{\mathrm{GD}}$ and compensation of the universal $\tau_{c c}$. The above statements contain a paradox because our ability to measure one of these quantities requires exact knowledge of the other.

The first observation of attosecond pulse structures in 2001 [24] relied on subtraction of $\tau_{\theta}$ computed for argon within the single-active electron approximation (SAE) using the potential proposed by Muller [62]. In Fig. 1.7 (a) we show the cross-section for ionization from the $3 p$ subshell in argon by absorption of one XUV photon for both the Muller potential (o) and using 
the Random-Phase Approximation with Exchange ( $\operatorname{RPAE}{ }^{7}$ ) for many-body screening effects $(+, \times, *)[63]$. Each model reproduces a Cooper minimum in the photoionization cross section [64] in qualitative agreement with the experimental total cross-section determined by Samson and Stolte [60]. The RPAE result, which includes ground-state correlation, is in excellent agreement with the measurement close to the ionization threshold. At higher energies the total cross-section differs from the partial RPAE cross-section due to opening of other ionization channels. In Fig. 1.7 (b) we show the corresponding atomic delays (computed from two-photon matrix elements) for argon within the SAE (o) and for RPAE screened XUV photon $(+, \times, *)$. The SAE agrees qualitatively with the correlated calculations, but it can hardly be used for calibration of the experimental data on the attosecond time scale.

In Fig. 1.7 (c) and (d) we show the partial cross-section and atomic delays for photoionization from the $3 s$ subshell of argon, respectively [63]. Interorbital correlation effects $(+, *)$ give rise to a minimum in the partial crosssection, as first pointed out by Amusia [65], and to a large positive peak in the atomic delay [12] that is not present for models which only include intra-shell correlation $(x)$. In Fig. 1.7 (c) the experimental measurements of the partial photoionization cross section by Möbus and co-workers [61] is in qualitative agreement with the RPAE result $(+, *)^{8}$. By subtraction of the data in Fig. 1.7 (b) and (d), an atomic delay difference of $\sim 25$ as is found at sideband $24(\sim 37.2 \mathrm{eV})$ between the $3 p$ and $3 s$ subshells. Unfortunately, this value does not agree well with the experimental measurements of $\sim 100$ as performed at Lund University $[10,12]$.

While the long-range phase, $\eta_{\mathrm{LR}}(x)$ in Eq. (1.2), is known from early quantum scattering theory [66], the accurate determination of the short-range phase, $\eta_{\mathrm{SR}}$, presents a numerical challenge for complex systems due to correlation effects [67]. Multi-electron screening effects on the delay in photoionization were first studied using the state-specific expansion approach (SSEA) [9] and by the RPAE for absorption of a single XUV photon $[68,69]$. Further works have included the multi-configurational Hartree-Fock method (MCHF) [51] and the time-dependent local density approximation (TDLDA) [70].

It was soon argued that the IR probe field should affect the photoionization delay measurement due to coupling to the long-range Coulomb potential $[10,36,38,49,58]$. Correlation effects in two-color fields (XUV $+\mathrm{IR})$ has been benchmarked for helium [46]. Approximate methods for larger atoms include the time-dependent $R$-matix [71], effective two-electron models [72], diagrammatic perturbation theory $[37,63]$ and application of the $B$-spline $R$-matrix (BSRM) for inclusion of shake-up processes [73].

Despite all this theoretical activity, the experimentally measured neon delay of $21 \pm 5$ as between the $2 p$ and $2 s$ subshell at an XUV photon energy of

\footnotetext{
${ }^{7}$ For details about the RPAE theory see Ref. [28].

${ }^{8}$ Surprisingly, the inclusion of correlation with the $L$-shell $(*)$ brings the calculation further away from the experimental measurements.
} 
$\sim 100 \mathrm{eV}[9]$ remains larger than the simulated values. The experimentally measured argon delay of $\sim 100$ as between the $3 p$ and $3 s$ subshell at an XUV excitation energy of $\sim 37 \mathrm{eV}[10,12]$ has been best reproduced by MCHF [51] and TDLDA calculations [70]. It has been found by Dixit and co-workers that the TDLDA method, in contrast to the RPAE model, produces a negative delay peak from the $3 s$ subshell [70]. A further study of the correlation effects by diagrammatic methods has confirmed that the sign of the delay peak depends on the details of the correlation model [63], but further work is needed to pin-point important interactions beyond the RPAE that include virtual shakeup (two holes and one electron) and knock-out (one hole and two electrons) processes.

We predict that in the coming years, the interpretation of attosecond experiments will offer a new testing ground for many-body calculations. Future experiments will provide interesting opportunities to test various theoretical methods for light-induced electron-electron interactions in connection to measurements of phases and delays in complex atomic and molecular systems. In the next section we provide an overview of how the paradox of attosecond delays was overcome experimentally by performing relative delay measurements from different initial states of the target.

\subsection{Review of experimental delay measurements}

The first experimental observations of photoionization time delays were made with the same experimental setups as those used for the characterization of attosecond pulses, but with one important modification: In these relative delay experiments, two photoelectron spectrograms were recorded simultaneously from two different initial states of the target $[6,9-12]$. Because it was the same attosecond pulses that triggered ionization from both initial states, the unknown exact shape of the attosecond pulses canceled out and the paradox of delays was circumvented. In these measurements photoelectron 'replicas' of the attosecond pulses are generated at different average kinetic energies corresponding to each initial state, say $A$ and $B$. These replicas were then characterized by using the IR-field as an ultrafast phase-gate so that the relative atomic delay between the two states could be observed: $\Delta \tau_{\theta}(A, B)=\tau_{\theta}(A)-\tau_{\theta}(B)$. Alternatively, if the stability of the attosecond pulses is good enough, the relative atomic delay can be studied sequentially in different target systems, thus revealing differences in the atomic delays associated with the two systems [7].

The experimental work on photoionization time-delays has been carried out on many-electron systems: condensed matter [6], noble gas atoms $[9,10,74]$ and molecules [7].

In the following, we will proceed with a brief overview of the current experimental efforts, adapted and extended from the corresponding section out of Ref. [13]. We will only review work on time-delay and phase measurements using attosecond pulse trains (APT) where we have taken an active part. 


\subsubsection{Atomic-delay measurements using attosecond pulse trains}

In an experiment conducted by Klünder and co-workers using an APT with a photon energy of $\sim 35 \mathrm{eV}[10]$, two RABITT scans were recorded simultaneously by ionizing both the $3 p$ and $3 s$ orbitals of argon. A schematic illustration of the experiment is shown in Fig. 1.8. As explained above, the unknown temporal structure of the attosecond pulses is subtracted without ever knowing the exact shape. The result is a relative delay of $\sim 100$ as between the ionization from the $3 p$ and $3 s$ subshells at the same ionizing photon energy. A large part of this measured delay was attributed to $\tau_{c c}$, see Fig. 1.6 (b), due to the different final energies of the photoelectrons originating from the two different subshells. In this work, the high-order harmonic comb was first

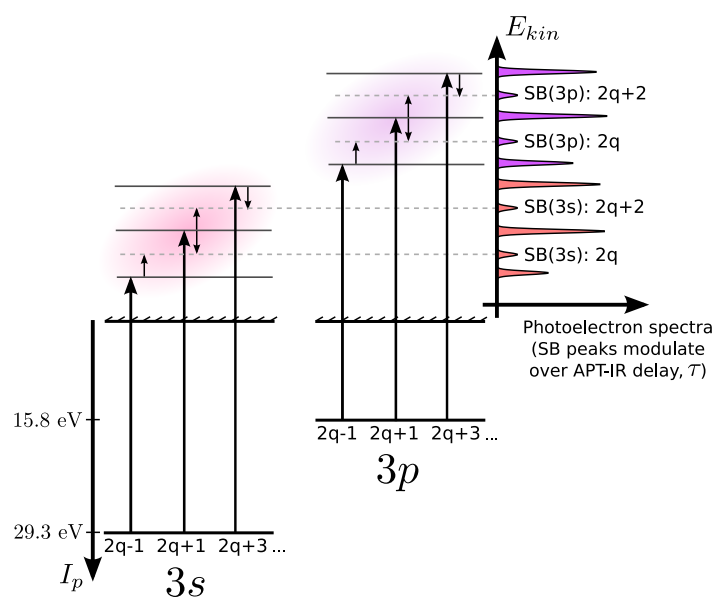

Fig. 1.8. Delay experiment for the $3 s$ and $3 p$ shells in Ar using APT: The APT is composed of three odd harmonics, $2 q+1$, that ionize (pump) electrons from either initial shell, $3 s$ or $3 p$, into the continuum. After absorbing one harmonic photon, the photoelectron can either absorb or emit a laser probe photon in order to reach an even number sideband (SB) state, labelled by $2 q$. The SB probability oscillates with the delay APT-IR delay, $\tau$, due to interference between the two quantum paths. Information about the attosecond timing is found in the relative modulation offset between the same sideband numbers from different initial states [10]. Figure adapted from Ref. [35].

passed through a thin chrome foil acting as a band-pass to separate four odd harmonics (21-27) of the 800-nm driving laser. In this way, only three sidebands, SB: 22, 24, 26, were produced when ionizing argon atoms from either orbital. This truncation of the harmonic comb was made to prevent different sidebands from different orbitals to overlap in photoelectron kinetic energy.

The analysis of the experiment [10] was first carried out within the SAE approximation using Hartree-Fock phase-shifts from the literature [75]. As 
shown in Fig. 1.7 (c)-(d), the photoelectrons from the inner subshell, $3 s$, couple strongly with the other electrons, and the corresponding dynamics is greatly altered due to correlation. This important effect was identified by Kheifets by considering RPAE effects in the absorption of a single XUV-photon [69].

The influence of a narrower atomic resonance has been observed experimentally by Swoboda and co-workers [8]. Fig. 1.9 illustrates the principle of the experiment. Here, an APT has the particular advantage that the discrete harmonics to which it corresponds in the spectral domain can be tuned towards a specific energy region of interest. The frequency of the harmonics from the HHG process can be tuned naturally by changing the fundamental (driving) laser pulse frequency, $\omega+\delta \omega$, so that the high-order harmonic frequencies increase or decrease by $\delta \Omega=(2 q+1) \delta \omega$. It was found that the phase of the modulation of the lowest-lying sideband in Helium depended critically on the frequency of the harmonics.

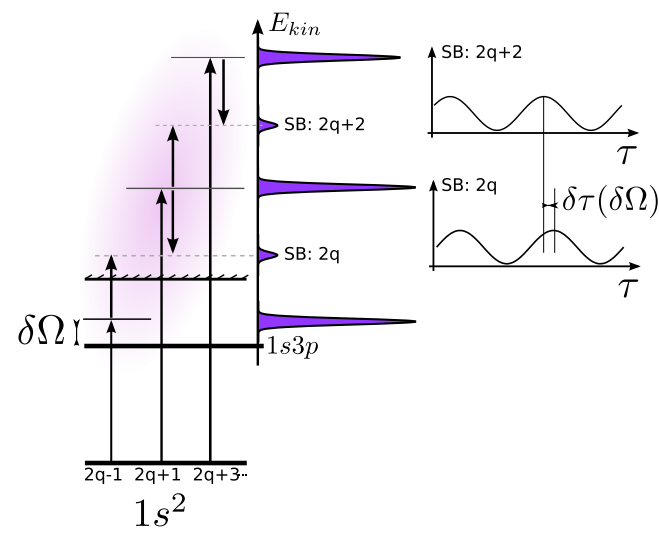

Fig. 1.9. Phase induced by the $1 s 3 p$ state in He using APT: The phase in photoionization, $\delta \tau$, depends on the detuning, $\delta \Omega$, between the harmonic, $(2 q-1) \omega=15 \omega$, and the bound state, $1 s 3 p$. The effect arises from the $\pi$-jump that occurs in the two-photon amplitude, when passing the resonance. The higher-lying sideband, SB: $2 q+2$, acts as an important "reference clock" in this experiment, which is mostly insensitive to small frequency changes of the harmonic fields [8]. Figure adapted from Ref. [35].

The observed modulation-shifts were attributed to a phase jump occurring when a below-threshold harmonic, $15 \omega<I_{p}^{(H e)}$, was scanned over the intermediate "resonant" bound state, $1 s 3 p{ }^{1} P_{1}$. By tuning the harmonic over the sharp resonance, an abrupt $\pi$-shift is expected. The experimental $\pi$-shift is "smoothed" due to the finite duration of the laser probe field and of the APT ( $~ 30 \mathrm{fs}$ ), which is much shorter than the resonance lifetime. In order to fully resolve the abrupt rise of the delay due to pure atomic effects, one needs 
harmonics that are narrower in frequency than the atomic resonance, i.e. an APT which is longer that the resonance lifetime.

A similar situation was observed by Haessler and co-workers with a shortlived complex continuum resonance in nitrogen molecules $[7,34]$ covered by harmonic 11 of the $800-\mathrm{nm}$ pump laser. Its lifetime was more comparable to the duration of the APT, implying that the observed modulation-shifts contain some information about the resonance lifetime. The experiment, very similar to the scheme shown in Fig. 1.9 but with the resonance situated just above the ionization threshold, compared ionization from the HOMO and the HOMO1 of nitrogen (corresponding to ions in their ground or first excited state, respectively), with additional resolution of vibrational states of the created ions, enabled by the relatively narrowband harmonics. As an additional "external" reference, the corresponding measurements were also made ionizing the $3 p$-orbitals of argon atoms, where the harmonic instead directly creates a photoelectron, $11 \omega>I_{p}^{(A r)}$, and where resonances do not contribute considerably.

The resonant channel involved in the nitrogen ionization starts by promoting an electron from the HOMO-2 to a state of auto-ionizing character from where it is ejected when its corresponding hole is filled by an electron from the HOMO. At the resonant XUV-photon energy, ionization to the ionic ground state is thus dominated by this resonant channel - at least in excited vibrational states, which are less efficiently populated by direct ionization from the HOMO. In the experiment, a large $0.9 \pi$-modulation-shift of sideband 12 was observed for a single detuning $\delta \Omega$. The accompanying theoretical study, including numerical ToF simulations like those in section 1.2, showed that the derivative of the modulation phase shift with respect to the detuning would give a physically representative Wigner-like delay for the two-photon XUV +IR ionization channel involving the resonance, reaching values of several femtoseconds. Interestingly, it is close to the lifetime of the resonance, suggesting a chronology (the electron spends a few femtoseconds in the intermediate resonance before auto-ionization and interaction with the IR field) in an a-priori non-sequential two-photon ATI transition. Note that this delay for the twophoton transition naturally includes the continuum-continuum delay, which is here not to be considered a "measurement-induced artefact" but part of the studied ionization dynamics.

Recently, Månsson and co-workers studied non-sequential double-ionization of xenon atoms [74]. They compare, at a total absorbed energy of $40.1 \mathrm{eV}$ (sideband 26), the reference, single ionization of the xenon $5 p$ state, with the non-sequential double-ionization channel leading to the ${ }^{1} D_{2}$ excited state of doubly-charged xenon ions, measured in parallel by detecting the two released photo-electrons in coincidence. This experimental work is important because it represents a way to study the phase of two-electron wave packets. 


\subsection{Conclusions}

In this chapter, we have illustrated how subtle short-range distorsions in atomic and molecular potentials can lead to variations in photoionization delays photoionization delays on the attosecond and femtosecond time scale. Such delays are nowadays measurable in experiments using two-color (XUVIR) laser fields. The time-domain perspective opened by such studies provides possibly more intuitive physical pictures as compared to the analytical derivations of stationary state phase shifts. Thus, exploring the time domain brings a complementary view from the traditional frequency domain that prevails in standard spectroscopy.

\section{Acknowledgement}

We acknowledge that elements of this chapter are reproduced from refs. [13, $63]$.

\section{References}

1. P. Agostini, L.F. DiMauro, Rep. Progr. Phys. 67(6), 813 (2004)

2. P.B. Corkum, F. Krausz, Nature Phys. 3(6), 381 (2007)

3. F. Krausz, M. Ivanov, Rev. Mod. Phys. 81(1), 163 (2009)

4. F. Lepine, M.Y. Ivanov, M.J.J. Vrakking, Nature Phot. 8(3), 195 (2014)

5. S.R. Leone, C.W. McCurdy, J. Burgdorfer, L.S. Cederbaum, Z. Chang, N. Dudovich, J. Feist, C.H. Greene, M. Ivanov, R. Kienberger, U. Keller, M.F. Kling, Z. Loh, T. Pfeifer, A.N. Pfeiffer, R. Santra, A. Schafer, K. Stolow, U. Thumm, M.J.J. Vrakking, Nature Phot. 8(3), 162 (2014)

6. A. Cavalieri, N. Müller, T. Uphues, V. Yakovlev, A. Baltuška, B. Horvath, B. Schmidt, L. Blümel, R. Holzwarth, S. Hendel, et al., Nature 449(7165), 1029 (2007)

7. S. Haessler, B. Fabre, J. Higuet, J. Caillat, T. Ruchon, P. Breger, B. Carré, E. Constant, A. Maquet, E. Mével, et al., Phys. Rev. A 80(1), 011404 (2009)

8. M. Swoboda, T. Fordell, K. Klünder, J. Dahlström, M. Miranda, C. Buth, K. Schafer, J. Mauritsson, A. LHuillier, M. Gisselbrecht, Phys. Rev. Lett. 104(10), 103003 (2010)

9. M. Schultze, M. Fieß, N. Karpowicz, J. Gagnon, M. Korbman, M. Hofstetter, S. Neppl, A. Cavalieri, Y. Komninos, T. Mercouris, et al., Science 328(5986), 1658 (2010)

10. K. Klünder, J. Dahlström, M. Gisselbrecht, T. Fordell, M. Swoboda, D. Guénot, P. Johnsson, J. Caillat, J. Mauritsson, A. Maquet, et al., Phys. Rev. Lett. 106(14), 143002 (2011)

11. S. Neppl, R. Ernstorfer, E.M. Bothschafter, A.L. Cavalieri, D. Menzel, J.V. Barth, F. Krausz, R. Kienberger, P. Feulner, Phys. Rev. Lett. 109, 087401 (2012)

12. D. Guénot, K. Klünder, C. Arnold, D. Kroon, J. Dahlström, M. Miranda, T. Fordell, M. Gisselbrecht, P. Johnsson, J. Mauritsson, et al., Phys. Rev. A 85(5), 053424 (2012) 
13. J. Dahlström, A. LHuillier, A. Maquet, J. Phys. B 45(18), 183001 (2012)

14. R. Pazourek, S. Nagele, J. Burgdörfer, Faraday Discuss. 163, 353 (2013)

15. E. Goulielmakis, M. Schultze, M. Hofstetter, V. Yakovlev, J. Gagnon, M. Uiberacker, A. Aquila, E. Gullikson, D. Attwood, R. Kienberger, et al., Science 320(5883), 1614 (2008)

16. D.H. Ko, K.T. Kim, J. Park, J.h. Lee, C.H. Nam, New. J. Phys. 12(6), 063008 (2010)

17. M. Drescher, M. Hentschel, R. Kienberger, M. Uiberacker, V. Yakovlev, A. Scrinzi, T. Westerwalbesloh, U. Kleineberg, U. Heinzmann, F. Krausz, Nature 419(6909), 803 (2002)

18. L. Argenti, C. Ott, T. Pfeifer, F. Martín, J. Phys.: Conference Series 488(3), 032030 (2014)

19. C. Ott, A. Kaldun, P. Raith, K. Meyer, M. Laux, J. Evers, C.H. Keitel, C.H. Greene, T. Pfeifer, Science 340(6133), 716 (2013)

20. E.P. Wigner, Phys. Rev. 98, 145 (1955)

21. F.T. Smith, Phys. Rev. 118(1), 349 (1960)

22. C.A. de Carvalho, H.M. Nussenzveig, Phys. Rep. 364(2), 83 (2002)

23. D.J. Tannor, Introduction to quantum mechanics - A time-dependent perspective (University Science Books, Sausalito CA, 2007)

24. P.M. Paul, E.S. Toma, P. Breger, G. Mullot, F. Augé, P. Balcou, H.G. Muller, P. Agostini, Science 292(5522), 1689 (2001)

25. Y. Mairesse, A. de Bohan, L.J. Frasinski, H. Merdji, L.C. Dinu, P. Monchicourt, P. Breger, M. Kovacev, R. Taïeb, B. Carré, H.G. Muller, P. Agostini, P. Salières, Science 302(5650), 1540 (2003)

26. Y. Mairesse, F. Quéré, Phys. Rev. A 71(1), 011401 (2005)

27. H. Park, R.N. Zare, J. Chem. Phys. 104, 4554 (1996)

28. M. Amusia, Atomic photoeffect (Plenum Press, New York, 1990)

29. M. Vacher, A. Maquet, R. Taïeb, J. Caillat, in preparation

30. F. Gel'mukhanov, H. Ågren,

31. H.G. Muller, Appl. Phys. B 74(1), s17 (2002)

32. W. Boutu, S. Haessler, H. Merdji, P. Breger, G. Waters, M. Stankiewics, L.J. Frasinski, R. Taïeb, J. Caillat, A. Maquet, P. Monchicourt, B. Carré, P. Salières, Nature Phys. 4, 545 (2008)

33. S. Haessler, J. Caillat, W. Boutu, C. Giovanetti-Teixeira, T. Ruchon, T. Auguste, Z. Diveki, P. Breger, A. Maquet, B. Carré, et al., Nature Phys. 6(3), 200 (2010)

34. J. Caillat, A. Maquet, S. Haessler, B. Fabre, T. Ruchon, P. Salières, Y. Mairesse, R. Taïeb, Phys. Rev. Lett. 106(9), 093002 (2011)

35. J. Dahlström, A. L'Huillier, J. Mauritsson, J. Phys. B 44(9), 095602 (2011)

36. J.M. Dahlström, D. Guénot, K. Klünder, M. Gisselbrecht, J. Mauritsson, A. LHuillier, A. Maquet, R. Taïeb, Chem. Phys. 414, 53 (2013)

37. J.M. Dahlström, T. Carette, E. Lindroth, Phys. Rev. A 86, 061402 (2012)

38. C.H. Zhang, U. Thumm, Phys. Rev. A 82(4), 043405 (2010)

39. R. Gaillac, A. Maquet, R. Taïeb, J. Caillat, in preparation

40. W.H. Press, S.A. Teukolsky, W.T. Vetterling, B.P. Flannery, Numerical Recipes: The Art of Scientific Computing (Cambridge University Press, 2007)

41. K.C. Kulander, K.J. Schafer, J.L. Krause, in Atoms in Intense Laser Fields, ed. by M. Gavrila (United States: Academic Press Inc, 1992)

42. P. Kruit, F. Read, J. Phys. E: Scientific Instruments 16(4), 313 (1983) 
43. A.T. Eppink, D.H. Parker, Review of Scientific Instruments 68(9), 3477 (1997)

44. J. Itatani, F. Quéré, G.L. Yudin, M.Y. Ivanov, F. Krausz, P.B. Corkum, Phys. Rev. Lett. 88(17), 173903 (2002)

45. F. Quéré, Y. Mairesse, J. Itatani, J. Mod. Opt. 52(2), 339 (2005)

46. R. Pazourek, J. Feist, S. Nagele, J. Burgdörfer, Phys. Rev. Lett. 108, 163001 (2012)

47. M. Chini, S. Gilbertson, S.D. Khan, Z. Chang, Opt. Expr. 18(12), 13006 (2010)

48. V. Véniard, R. Taïeb, A. Maquet, Phys. Rev. A 54(1), 721 (1996)

49. S. Nagele, R. Pazourek, J. Feist, K. Doblhoff-Dier, C. Lemell, K. Tőkési, J. Burgdörfer, J. Phys. B 44(8), 081001 (2011)

50. S. Nagele, R. Pazourek, J. Feist, J. Burgdörfer, Phys. Rev. A 85(3), 033401 (2012)

51. T. Carette, J.M. Dahlström, L. Argenti, E. Lindroth, Phys. Rev. A 87, 023420 (2013)

52. L.B. Madsen, Am. J. Phys. 73(1), 57 (2005)

53. A. Maquet, R. TaÏeb, J. Mod. Opt. 54(13-15), 1847 (2007)

54. A.J. Galn, L. Argenti, F. Martín, New J. Phys. 15(11), 113009 (2013)

55. V.S. Yakovlev, J. Gagnon, N. Karpowicz, F. Krausz, Phys. Rev. Lett. 105(7), $073001(2010)$

56. C.H. Zhang, U. Thumm, Phys. Rev. A 84(3), 033401 (2011)

57. O. Smirnova, M. Spanner, M.Y. Ivanov, J. Phys. B 39(13), S323 (2006)

58. M. Ivanov, O. Smirnova, Phys. Rev. Lett. 107(21), 213605 (2011)

59. Q.C. Ning, L.Y. Peng, S.N. Song, W.C. Jiang, S. Nagele, R. Pazourek, J. Burgdörfer, Q. Gong, Phys. Rev. A 90, 013423 (2014)

60. J. Samson, W. Stolte, J. Electron. Spectrosc. Relat. Phenom. 123(23), 265 (2002)

61. B. Möbus, B. Magel, K.H. Schartner, B. Langer, U. Becker, M. Wildberger, H. Schmoranzer, Phys. Rev. A 47, 3888 (1993)

62. H.G. Muller, Phys. Rev. A 60, 1341 (1999)

63. J.M. Dahlström, E. Lindroth, J. Phys. B 47(12), 124012

64. J.W. Cooper, Phys. Rev. 128(2), 681 (1962)

65. M. Amusia, V. Ivanov, N. Cherepkov, L. Chernysheva, Phys. Lett. A 40(5), 361 (1972)

66. L.D. Landau, E.M. Lifshitz, Quantum Mechanics (Non-Relativistic Theory) (Pergamon Press, 1965)

67. K. Chadan, P.C. Sabatier, Inverse Problems in Quantum Scattering Theory (New York: Springer, 1989)

68. A. Kheifets, I. Ivanov, Phys. Rev. Lett. 105(23), 233002 (2010)

69. A.S. Kheifets, Phys. Rev. A 87, 063404 (2013). DOI 10.1103/PhysRevA.87.063404

70. G. Dixit, H.S. Chakraborty, M.E.A. Madjet, Phys. Rev. Lett. 111, 203003 (2013)

71. L. Moore, M. Lysaght, J. Parker, H. van der Hart, K. Taylor, Phys. Rev. A 84(6), 061404 (2011)

72. S. Nagele, R. Pazourek, J. Feist, J. Burgdörfer, Phys. Rev. A 85, 033401 (2012)

73. J. Feist, O. Zatsarinny, S. Nagele, R. Pazourek, J. Burgdörfer, X. Guan, K. Bartschat, B.I. Schneider, Phys. Rev. A 89, 033417 (2014)

74. E.P. Mansson, D. Guenot, C.L. Arnold, D. Kroon, S. Kasper, J.M. Dahlstrom, E. Lindroth, A.S. Kheifets, A. L/'Huillier, S.L. Sorensen, M. Gisselbrecht, Nature Phys. 10(3), 207 (2014) 
75. D.J. Kennedy, S.T. Manson, Phys. Rev. A 5(1), 227 (1972) 